\title{
Bistability and Pulsations in Semiconductor Lasers with Inhomogeneous Current Injection
}

\author{
CHRISTOPH HARDER, KAM Y. LAU, MEMBER, IEEE, AND AMNON YARIV, FELLOW, IEEE
}

\begin{abstract}
Bistability and pulsation at microwave frequencies are observed in $\mathrm{CW}$ GaAs semiconductor lasers with inhomogeneous current injection. Inhomogeneous current injection is achieved with a segmented contact structure. Crucial to the understanding of the characteristics of this device is the discovery of a negative differential electrical resistance across the contacts of the absorbing section. Depending on the electrical bias condition, this negative differential resistance leads to bistability or light-jumps and self pulsations. A simple model based on conventional rate equations with a linear gain dependence on carrier density explains the observed behavior and suggests a new mechanism in inhomogeneously pumped diode lasers for light-jumps and pulsations which does not depend on the condition for the usually proposed repetitively $Q$-switching. Investigation of the switching dynamics of this bistable optoelectronic device reveals a delay time which is critically dependent on the trigger pulse amplitude and typically on the order of a few nanoseconds with power-delay products of $100 \mathrm{pJ}$. The observed critical slowing down and its origin is discussed. We also report on the characteristic of this laser coupled to an external optical cavity and we demonstrate successfully that this bistable laser can be used as a self coupled stylus for optical disk readout with an excellent signal to noise ratio.
\end{abstract}

\section{INTRODUCTION}

B ISTABLE semiconductor lasers based on inhomogeneous current injection resulting from a segmented contact structure were proposed over fifteen years ago [1]. However, actual devices fabricated to date showed no or only a small hysteresis [2]-[6]. In addition, these devices were beset by pulsations of the optical output [6]-[10] for reasons not well understood at the time. Recently, we demonstrated a bistable buried heterostructure laser with a large hysteresis and no pulsations [11]-[13]. Crucial to the understanding of the behavior of this bistable laser was the discovery of a negative differential resistance across the absorber section. Depending on the electrical biasing source, this negative resistance leads to a) bistability with a very large hysteresis in the light-current characteristic with no self pulsations or b) a narrow hysteresis (or a light jump) and self pulsations. The negative resistance suggests a new mechanism for the observed self pulsations which does not depend on the condition for the usually proposed repetitively $Q$-switching [7], [14], [15]. Experiments reveal that this bistable laser switches from one state to the other after a delay time which is critically dependent on the

Manuscript received March 8,1982 . This work was supported by the Office of Naval Research, the National Science Foundation under the Optical Communication Program, and the Air Force Office of Scientific Research.

Ch. Harder and A. Yariv are with the California Institute of Technology, Pasadena, CA 91125.

K. Y. Lau is with the Ortel Corporation, Alhambra, CA 91803. trigger pulse overdrive. This critical slowing down [16], [17] of the delay time is discussed in some detail and a small signal interpretation of this phenomenon will be presented. In an analysis we will show that the observed complex behavior of a laser with inhomogeneous current injection can be explained with the conventional rate equations only when the electrical aspect of the laser diode is also taken into account. The electrical characteristic of the device also manifests a giant hysteresis which mimicks the optical hysteresis.

Even though this bistable laser switches within a few nanoseconds at power delay products of a few picojoules, we do not believe that this device will be able to compete with conventional logic in the near future. Its main application will be in the field of optoelectronic signal processing. We demonstrate successfully that this bistable laser can be used as an optical stylus for optical disk readout with an excellent signal to noise ratio (due to the bistable characteristic) and a large electrical output signal.

\section{Static Characteristic}

CW-GaAs buried heterostructure $(\mathrm{BH})$ lasers with a split contact on the top p-side as shown in Fig. 1 have been fabricated by a standard process in our laboratory [18] and typical dimensions and doping levels are given in the figure. The upper cladding layer of these BH lasers is only lightly p-doped in order to increase the lateral parasitic resistance $R_{p}$ between the two contact pads which is measured to be around $60 \mathrm{k} \Omega$. It will be shown later that a good electrical isolation between the two segments is essential for the operation of this laser as a bistable element. Before the devices are cleaved, a $25 \mu \mathrm{m}$ wide gap is etched into the p-contact metallization. Near- and farfield measurements show that the laser is always operating in the fundamental transverse mode, demonstrating the effectiveness of the dielectric waveguide. The BH structure thus makes it possible to isolate and study the effects of inhomogeneous excitation with a device whose electronic and optical properties are stable and simple.

If both sections are connected together, that is, under homogeneous excitation, the light-current characteristic displays the conventional linear behavior above threshold as shown in Fig. 2, curve (a). For bistable operation, the absorber section (the section which is $100 \mu \mathrm{m}$ long) is pumped with a constant current $I_{2}=-110 \mu \mathrm{A}$. The light output is shown in Fig. 2, curve (b) as a function of $I_{1}$, the current through the gain section. This characteristic displays a large hysteresis extending from $I_{1}=30 \mathrm{~mA}$ (the switch-on current) to $I_{1}=22 \mathrm{~mA}$ (the switchoff current). The characteristic is also shown for $I_{2}=-100 \mu \mathrm{A}$, 


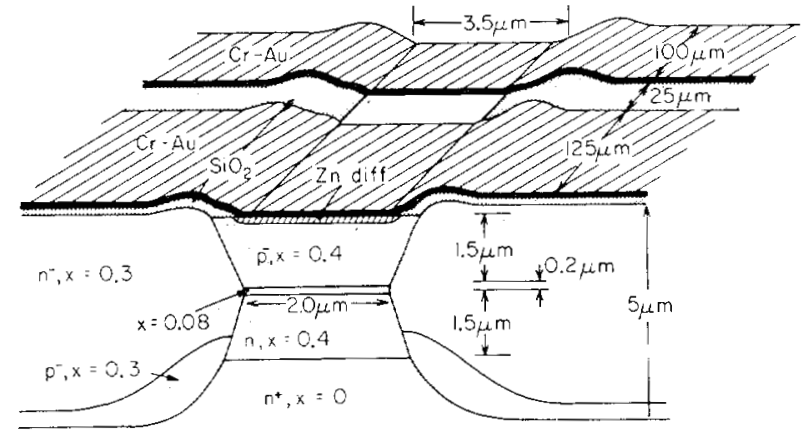

Fig. 1. $\mathrm{Ga}_{1}-x \mathrm{Al}_{x}$ As buried heterostructure laser with a segmented contact.

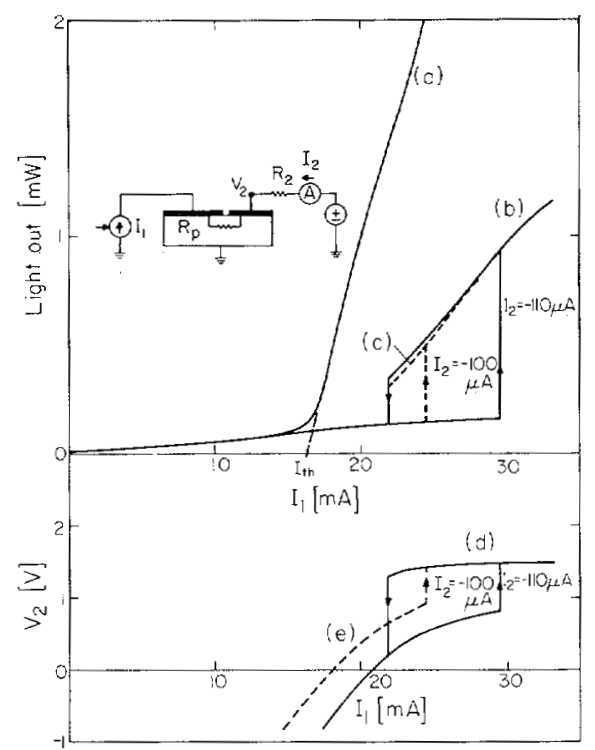

Fig. 2. Measured static characteristic of the two segment contact laser. The top half shows the light-current characteristic. For homogeneous pumping: curve (a). $I_{\text {th }}$ is the conventional threshold current. For inhomogeneous pumping: curve (b) for $I_{2}=-110 \mu \mathrm{A}$ and curve (c) for $I_{2}=-100 \mu \mathrm{A}$. The parasitic resistance is $R_{p}=60 \mathrm{k} \Omega$ and the current source impedance is $R_{2}=400 \mathrm{k} \Omega$. The lower half shows the voltage $V_{2}$ across the absorber section as function of $I_{1}$, the current through the gain section for $I_{2}=-110 \mu \mathrm{A}$ [curve (d)] and for $I_{2}=$ $-100 \mu \mathrm{A}$ [curve (e)].

curve (c) and the sensitivity of the size of the hysteresis on the amount of saturable absorption (i.e., $I_{2}$ ) can be seen. Note, that $I_{2}$ is negative, that is, carriers are extracted from the absorber section.

In the lower half of Fig. 2 the voltage $V_{2}$ across the absorbing section is shown [curve (d) and curve (e)] as a function of $I_{1}$. The main feature of this characteristic is that when the device is lasing (when the absorption is bleached) the voltage $V_{2}$ is clamped at $1.45 \mathrm{~V}$, the bandgap voltage of $\mathrm{GaAs}$. This demonstrates the one to one correspondence of the voltage across the contacts, the quasi-Fermi level separation and the carrier concentration in the active region.

In Fig. 3 the current-voltage characteristic $\left(I_{2}-V_{2}\right)$ of the absorbing section is shown for different currents $I_{1}$ through the gain section. The negative differential resistance in this characteristic is of particular interest and deserves special attention. This negative differential resistance is, as will be shown, optoelectronic in origin. The mechanism causing it can be understood with the following simple model as illus-

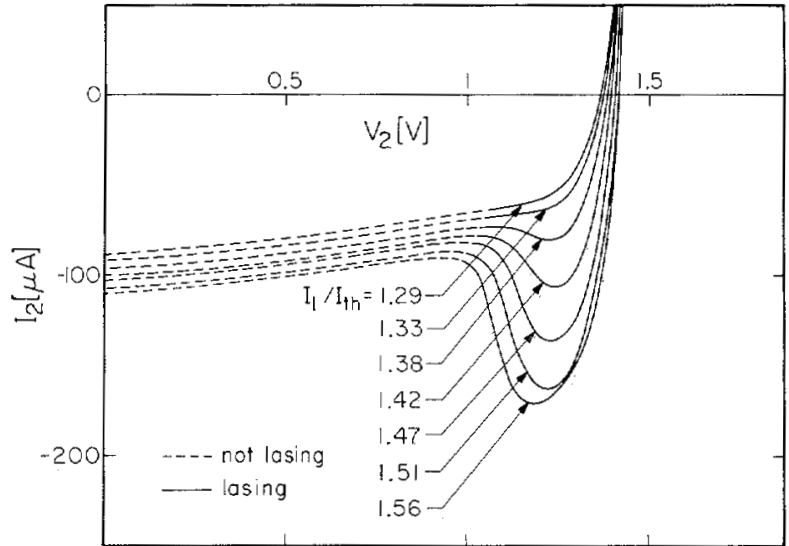

Fig. 3. Current-voltage $\left(I_{2}-V_{2}\right)$ characteristic of the absorber section for different currents $I_{1}$ through the gain section. $I_{\text {th }}$ is defined in Fig. 2.

trated in Fig. 4. Consider the bistable laser consisting of two parts, a gain section which is pumped with a constant current $I_{1}$ and an absorber section which acts like a photodiode within the optical cavity. The current $I_{2}$ through this photodiode consists of two terms: the first one is the normal diode current which depends exponentially on the applied voltage $V_{2}$ while the second term corresponds to a negative photo induced current $I_{P h}$ which is proportional to the photon density in the active region. (These photons are generated under the gain contact and guided to the absorbing region under the second contact). This photodiode characteristic is drawn on the right side of Fig. 4 for eight different normalized photon densities, $P=0$ to $P=7$. We shall now show how the measured $I_{2}-V_{2}$ characteristic is produced. For zero voltage $V_{2}$ the absorption of the photodiode is strong, thus suppressing stimulated emission and only a small photocurrent due to spontaneous emission in the gain section is generated. Increasing the voltage $V_{2}$ reduces the absorption, thus favoring stimulated emission which increases the photon density, generating a larger negative photo current in the absorbing diode. Increasing $V_{2}$ further increases the negative photo current $I_{P h}$, thus producing the negative slope. Finally, at large voltages $V_{2}$, the positive exponential term representing the normal diode behavior dominates and $I_{2}$ increases once more. This specific curve shown on the right side of Fig. 4 is obtained for a fixed gain current $I_{1}$. The whole set of curves as shown in Fig. 3 is obtained for different currents through the gain section.

\section{Bistability and Self-Puldsations}

We now show how the measured $I_{2}-V_{2}$ characteristic of the absorber section can lead under different biasing conditions to bistability or self-pulsation. The $I_{2}-V_{2}$ characteristic is shown again in Fig. 5 along with the characterization of the source driving this section, the load line. This line shows the voltage available to the absorber section as function of the current through it. The state of the system satisfying all static circuit equations is given by the intersection of the load line with the characteristic of the device. The state $P_{1}$ in Fig. 5 is the intersection of the absorber characteristic with the load line corresponding to a bias resistance of $R_{L}=20 \mathrm{k} \Omega$ and a current injection into the gain section of $I_{1} / I_{\mathrm{th}}=1,29$. In this 
Opto-Electronic Characteristics of a Bistable Laser

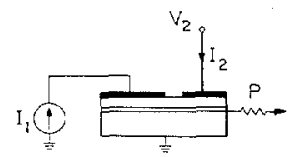

$$
\begin{aligned}
& \text { photodiode } \\
& I_{2}=I_{s}\left(e^{\frac{v_{z}}{u_{T}}}-1\right)-I_{P h} \\
& I_{P h}=\eta \cdot P
\end{aligned}
$$

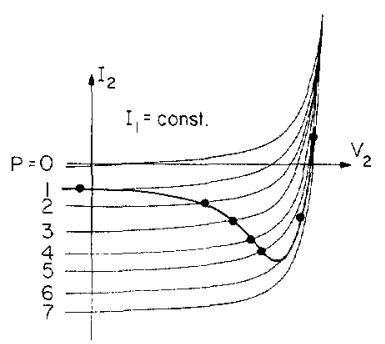

Fig. 4. Model to explain the current-voltage $\left(I_{2}-V_{2}\right)$ characteristic of the absorber section for a fixed current $I_{1}$ through the gain section. See text for explanation.

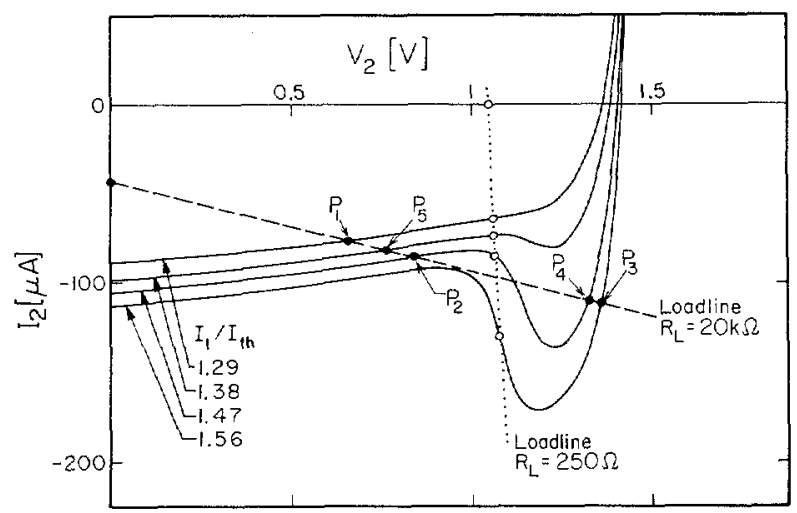

Fig. 5. Current-voltage $\left(I_{2}-V_{2}\right)$ characteristic of the absorber section for different currents $I_{1}$ through the gain section. Also shown are two load lines corresponding to a load resistance of $R_{L}=20 \mathrm{k} \Omega$ and $R_{L}=250 \Omega$.

state the laser is switched off. Increasing $I_{1}$ causes the intersection point to move along the load line from $P_{1}$ to $P_{2}$ and at $I_{1} / I_{\text {th }}=1.56$ to jump to $P_{3}$ since this is the only intersection of the load line with the $I_{2}-V_{2}$ characteristic. In this state the laser is switched on. A decrease of $I_{1}$ now causes the state to move back to $P_{4}$ and then to jump back to $P_{5}$, at which point the laser is switched off. A laser with such a large load resistance will display a hysteresis in the light versus current $I_{1}$ characteristic. This can also be seen from the fact that the load line has two stable intersections with the $I_{2}-V_{2}$ characteristic, e.g., for $I_{1} / I_{\mathrm{th}}=1.47$, the two stable states $P_{2}$ (switched off) and $P_{4}$ (switched on). The load line for a small resistance $R_{L}=250 \Omega$ is also shown in Fig. 5 , as the dotted line. This load line has always just one intersection with the characteristic of the device and the laser will consequently not display bistability. The laser with such a small load resistance can be biased to operate with its absorbing section in the regime of negative differential resistance. This leads to electrical microwave oscillation and concomittant light intensity pulsations.

The load resistance discussed above is essentially $R_{2}$ in parallel with $R_{p}, R_{L}=R_{p} R_{2} /\left(R_{p}+R_{2}\right)$ (see Fig. 2) and is always smaller than $R_{2}$ or $R_{p}$. Since bistable operation is only obtained with a large $R_{L}$, both the source resistance $R_{2}$ and the parasitic resistance $R_{p}$ have to be large. This is demonstrated in Fig. 6 where the light-current characteristic is shown for the two biasing situations discussed above, namely for a
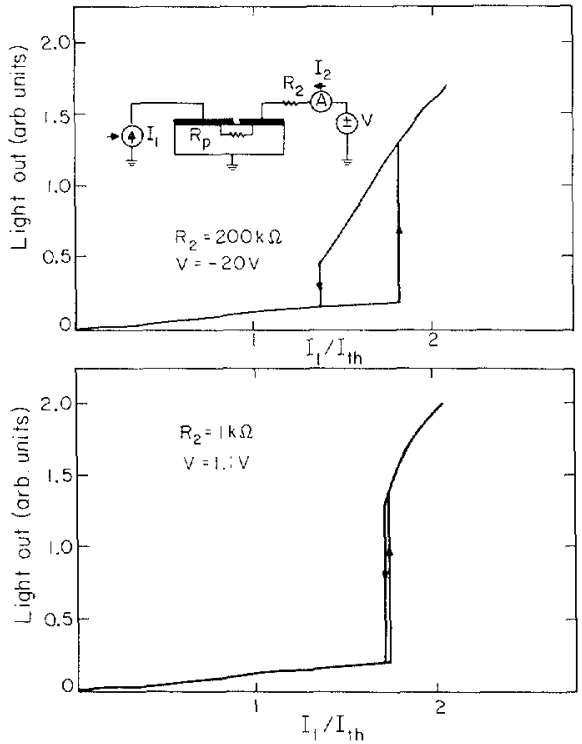

Fig. 6. Light-current characteristic for two different bias conditions of the absorber section. The characteristic in the upper half corresponds to biasing the absorber section with a large resistance (current biasing), in the lower half to biasing the absorber section with a small resistance (voltage biasing).

large $R_{L}=46 \mathrm{k} \Omega$ (top half) and a small $R_{L}=1 \mathrm{k} \Omega$ (bottom half). For a large $R_{L}$, the characteristic displays the predicted large hysteresis and the light output is stable, no pulsations can be observed within the detector bandwidth of $5 \mathrm{GHz}$. For a small $R_{L}$ the characteristic has only a very narrow hysteresis, a light jump, and the light output oscillates at microwave frequencies. Since the negative differential resistance is not frequency selective (at frequencies below a few $\mathrm{GHz}$ ) the device oscillates at the resonance of any frequency selective element coupled to the gain mechanism. Experiments show [6] that the device oscillates at the relaxation resonance frequency which is between $500 \mathrm{MHz}$ and $2 \mathrm{GHz}$ for this device depending on $I_{1}$. Our earlier lasers failed to produce a sizable hysteresis and were self pulsating [6] because their parasitic resistance (measured to be $1 \mathrm{k} \Omega$ ) was too small to allow bistable operation. The model described above could possibly explain a mechanism of self pulsations in aged lasers. Inhomogeneous excitation could be caused by degradation of the contacts or development of centers with enhanced nonradiative recombination. Note that these self pulsations are not due to a repetitively $Q$-switched mechanism, but are the result of the optoelectronically generated negative resistance and do not result, as will be shown in Section $\mathrm{V}$, from sublinear gain dependence.

In Fig. 7 the laser emission spectra are shown for the cases of bistable [Fig. 7(a)] and pulsating [Fig. 7(b)] operation. In the bistable case the laser light is emitted into one longitudinal mode and the linewidth is limited by the resolution of the $3 / 4$ $\mathrm{m}$ spectrometer to $0.2 \mathrm{~A}$. Under pulsating operating conditions, the spectrum breaks apart into some 10 longitudinal modes. Note that the linewidth of each of the longitudinal modes is broadened. This cannot be due to unresolved transverse modes since far-field and near-field measurements show that the laser is always operating in the fundamental transverse mode. The carrier concentration in the active region is oscillating with a 


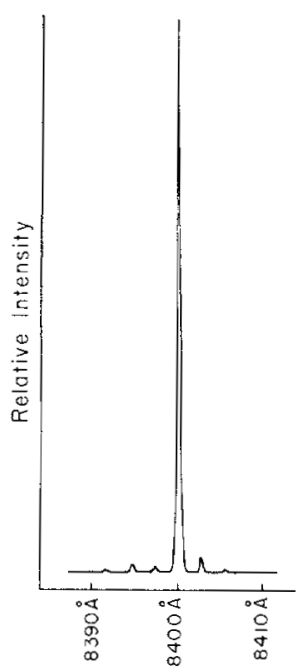

(a)

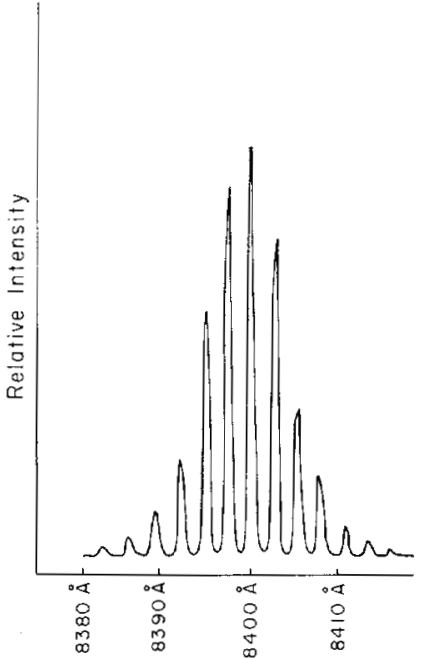

(b)
Fig. 7. Optical spectrum of the laser: under bistable operation (a), and under pulsating operation (b)

large amplitude during pulsations thus causing the index of refraction in the active region to change [19] resulting in a periodically pulling of the Fabry-Perot modes. This effect could explain the broadening of the observed time averaged spectrum.

\section{SwITchING}

The laser has two stable states when biased within the hysteresis loop. The laser can be switched off by increasing the absorption for a short time by some means. This can be achieved by either increasing the current $I_{1}$ through the gain section for a short time (gain-switching), by injecting a positive current pulse into the absorber section (absorber-switching) or by bleaching the absorber with an externally injected optical pulse (optical switching). In this section we discuss the dynamics of the gain switching, in particular the technologically important problem of delays between trigger pulse and switching. Such delays are observed in almost every bistable system including electronic Schmitt triggers and pure optical systems [16], [17], and it is well known that they can be reduced by increasing the trigger pulse amplitude. From a thermodynamic point of view the laser system undergoes a first order phase transition during the switching [20], [21]. As far as device applications are concerned, one of the most important predictions gained from this thermodynamic point of view is that of the critical slowing down, i.e., an extremely slow return of the system to the equilibrium or a very slow response time to a perturbation in the vicinity of the phase transition point [16], [17].

The delay between trigger pulse and switching of the laser can be very long compared to any physical lifetime associated with the system, such as electron-hole recombination or RCtime constant while the switching itself is relatively fast. For bistable operation the laser is biased at a constant current $I_{2}$ and at a current $I_{1}$ corresponding to the middle of the hysteresis loop, e.g., for the device shown in Fig. $2 I_{2}=-110 \mu \mathrm{A}$ and $I_{1}=26 \mathrm{~mA}$. A small positive current pulse superimposed on $I_{1}$ switches the laser to the high (lasing) state while a subsequent negative pulse switches the laser back to the low state

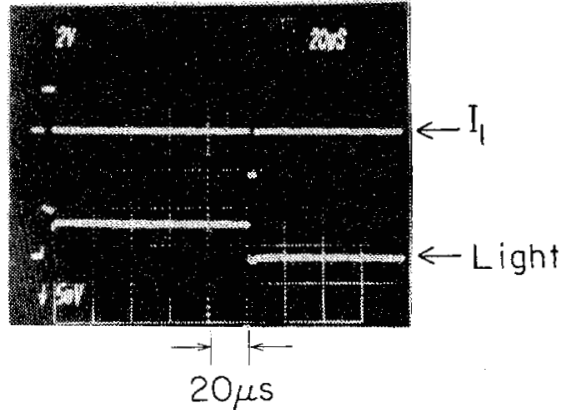

Fig. 8. Gain switching of the bistable laser. Top trace: Current $I_{1}$ through the gain section. Lower trace: Light output, Horizontal: $20 \mu \mathrm{s} / \mathrm{div}$.

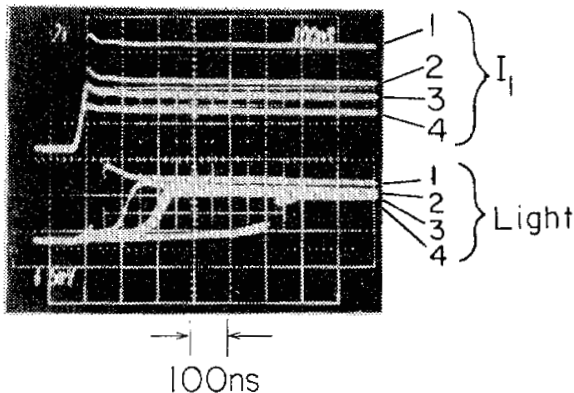

Fig. 9. Delay of light output for four different trigger pulse amplitudes. Horizontal: $100 \mathrm{~ns} / \mathrm{div}$.

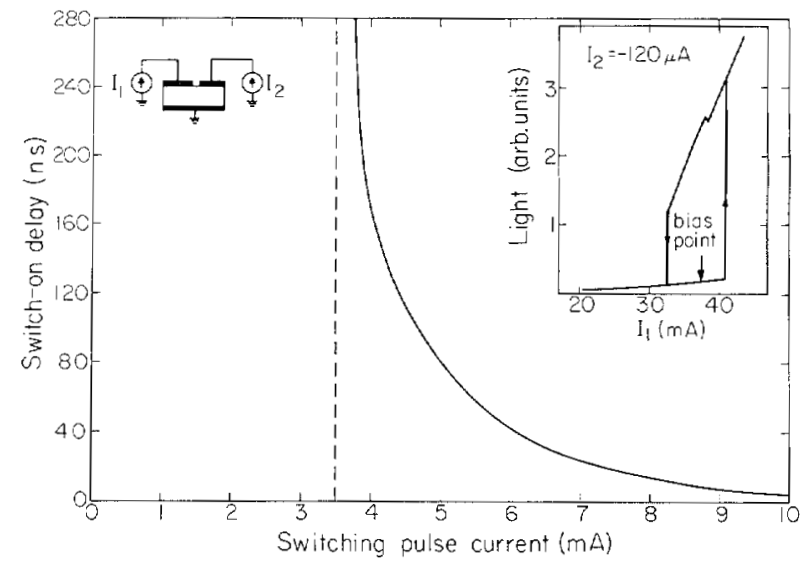

Fig. 10. Switch-on delay time as function of the trigger pulse amplitude. The critical slowing down of the switch delay occurs for pulse amplitudes of $3.5 \mathrm{~mA}$.

as shown in Fig. 8. A closer examination of the switch-on, as shown in Fig. 9, reveals a delay time that is dependent on the amount of trigger pulse overdrive. This delay time dependence on the switch pulse amplitude is shown in Fig. 10 and it can be divided into two regimes, a critical slowing down regime for small overdrive amplitudes and a noncritical regime for large overdrives. The following are the major features of the switching characteristic.

The critical regime is only observed for very small overdrives and is characterized by a dramatic increase of the switching delay up to several hundred nanoseconds. The behavior is that of the conventional critical slowing down. At higher drive currents, the switching delay is relatively noncritical and is the time required for the carrier density in the gain and absorber section to rise to the level where lasing can occur. This time 
lapse is in the order of a few carrier lifetimes. A similar delay occurs during switch on of common injection lasers [22].

The carrier lifetime in the gain section where the carrier density is high is in the order of a few nanoseconds, while that one in the low carrier density absorber section is in the tens of nanoseconds. The dominating time constant is, unfortunately, the long one. Fig. 9 shows multiple traces of the switching behavior for four different drive currents (labelled $I_{1}$ ). The lowest trace of $I_{1}$ is very close to the threshold for switching and the critical slowing down in the corresponding light output is evident. The delay can be reduced drastically to below 20 ns when the trigger current is increased to about $9 \mathrm{~mA}$. A switching delay of $5 \mathrm{~ns}$ can be achieved with a pulse of $10 \mathrm{~mA}$ which corresponds to an intrinsic power delay product of $100 \mathrm{pJ}$ for gain switching. While the switch-on shows these interesting effects, the switch-off is usually fast and without delay. This arises from the fact that the switch-off process involves stimulated recombination of carriers at high density which is extremely fast. To observe the inherent switching behavior, the external parasitic capacitance at the absorbing section must be reduced to a minimum, since a switching of the device is accompanied by a change of the voltage, and thus charge, across the absorber section as can be seen in Fig. 2. In our experiment the diode was isolated from the capacitance of the current source by placing a $250 \mathrm{k} \Omega$ resistance as close to the diode as possible.

Considerable insight into the switching dynamics can be gained by probing the voltages across the gain and absorber sections, thus in effect probing the carrier densities. These results are presented elsewhere [12]. Experiments on absorber switching (by injecting a current pulse into the absorber section) reveal a switch on delay in the hundreds of nanoseconds for moderate overdrives and of $20 \mathrm{~ns}$ for a large overdrive of $150 \mu \mathrm{A}$ corresponding to a power delay product of $6 \mathrm{pJ}$.

\section{Static Analysis}

The purpose of this section is to analyze a simple model which explains the main features of a semiconductor laser with inhomogeneous current injection along the active region. This model emphasizes the relevant mechanisms and parameters responsible for the bistability and the negative differential resistance across the absorbing section. First the light-current characteristic is calculated using familiar rate equations. When we add equations describing the electrical aspect of the device a negative differential resistance is predicted. To clarify the intrinsic frequency limitation of this negative differential resistance a small signal analysis is performed and an electrical equivalent circuit is developed.

A set of three rate equations is the starting point of this analysis, one for the density of minority carriers in the gain section, one for those in the absorbing section and a third one for the density of photons in the lasing mode. The confinement of the injected carriers in a buried heterostructure laser to the small dimensions of the active region $(2 \mu \mathrm{m} \times 0.2 \mu \mathrm{m})$ justifies the characterization of the inversions by their average over these dimensions. Measurements show that the built in dielectric waveguide forces the laser to operate under all con- ditions in the fundamental transwerse mode. In addition, when this laser is biased for stable operation, the light is emitted into one longitudinal mode. This simple optical behavior can be modeled by one rate equation for the density of photons in the lasing mode. The carrier and photon densities have been averaged over the length of the device to render the calculation tractable. This assumption is approximately correct at high photon densities when the absorber section is saturated and bleached. At low photon densities this assumption is violated due to superluminescent effects. The three equations are

$$
\begin{aligned}
\frac{d N_{1}}{d t}= & \frac{I_{1}}{e V_{1}}-B\left(N_{1}+N_{A}\right) N_{1}-A\left(N_{1}-N_{t r}\right) P \\
\frac{d N_{2}}{d t}= & \frac{I_{2}}{e V_{2}}-B\left(N_{2}+N_{A}\right) N_{2}-A\left(N_{2}-N_{t r}\right) P \\
\frac{d P}{d t}= & A\left(\alpha_{1} N_{1}+\alpha_{2} N_{2}-N_{t r}\right) P-\frac{P}{\tau_{P}} \\
& +\beta B\left\{\alpha_{1} N_{1}\left(N_{1}+N_{A}\right)+\alpha_{2} N_{2}\left(N_{2}+N_{A}\right)\right\} .
\end{aligned}
$$

$V_{1}=\alpha_{1} V$ is the volume of the active region with gain, $N_{1}$ is the inversion density of carriers in the gain region, and $I_{1}$ is the current injected into it. $V_{2}, \alpha_{2}, N_{2}$, and $I_{2}$, are the corresponding variables in the absorber section, $\left(\alpha_{1}+\alpha_{2}=1\right) . P$ is the photon density in the lasing mode, $e$ is the electronic charge, $\tau_{P}$ is the photon lifetime and $\beta$ is the coupling coefficient for spontaneous emission into the lasing mode. Since the carrier densities vary over a large range it is not reasonable to assume a constant spontaneous lifetime $\tau_{s}$. These rate equations incorporate a bimolecular recombination rate where $B$ is the recombination constant and $N_{A}$ is the doping concentration of the p-type background in the active region. A linear gain dependence on injected carrier density $G(N)=A\left(N-N_{t r}\right)$ is used in these calculations in agreement with recent measurements of the gain in undoped or only slightly doped GaAs [23]. The same coefficients are used for the gain and absorbing section assuming equal conditions such as temperature and material in both sections.

It is crucial to appreciate the importance of the functional dependence of the gain on carrier density $G(N)$ for the stability of a laser diode with inhomogeneous injection. It has been shown [7], [14], [15] that a sublinear gain dependence such as $G(N)=A\left(N-N_{t r}\right)^{\alpha}$ with $\alpha<1$, causes an inhomogeneously pumped laser to pulsate due to a repetitively Q-switched mechanism. On the other hand a superlinear gain dependence such as $G(N)=A\left(N-N_{t r}\right)^{\alpha}$ with $\alpha>1$, has a stabilizing effect, suppressing pulsations and bistability. A laser with a linear gain dependence $G(N)=A\left(N-N_{t r}\right)$ displays a more complex behavior. In the following analysis it will be shown that for such a gain dependence a laser with inhomogeneous injection can be made to display bistability or self pulsations depending on the electrical biasing circuit.

The following values of the various parameters have been used: $\alpha_{1}=0.5 ; \alpha_{2}=1-\alpha_{1}=0.5 ; V=250 \mu \mathrm{m} \times 2 \mu \mathrm{m} \times 0.2$ $\mu \mathrm{m} ; \quad B=3 \times 10^{10} \mathrm{~cm}^{3} / \mathrm{s} ; \quad N_{A}=3 \times 10^{17} \mathrm{~cm}^{-3} ; A=2.34 \times$ $10^{-6} \mathrm{~cm}^{3} / \mathrm{s}$ corresponding to an absorption coefficient of $\alpha=$ 
$-230 \mathrm{~cm}^{-1}$ at zero inversion; $N_{t r}=0.83 \times 10^{18} \mathrm{~cm}^{-3} ; \tau_{p}=1.5$ ps corresponding to waveguide and other distributed losses $\alpha=40 \mathrm{~cm}^{-1}$ and $\beta=10^{-4}$. To calculate the static light current characteristic, the time derivatives in (1) through (3) have been set to zero. The solution shown in Fig. 11 displays the main features of the measurement (Fig. 2), linear characteristic under homogeneous excitation, and a sizable hysteresis if the absorbing section is biased with a small negative current. Measured and calculated threshold currents agree if we take a current confinement factor of one half into account. Note that the calculation predicts a hysteresis for $I_{2}=0$. This is not observed, probably because the superradiance of the gain section is intense enough to saturate this weak absorption. Measurements reveal that the light coming out of the gain section facet is weaker than that from the absorber section [24]. The average light from both sides compares well with the calculated characteristic. The carrier densities in the gain section and absorber section are shown in Fig. 12 as function of $I_{1}$.

The rate equations (1) through (3) model the device with given currents $I_{1}$ and $I_{2}$ being injected into the gain and absorber sections and are therefore sufficient for a complete description if the gain and absorber section are biased with sources whose equivalent impedance is much larger than the impedance of the gain and absorber section. Although this condition is always fulfilled in the case of the gain section, it is violated for the absorbing section which is typically only slightly forward biased.

The external voltage $V_{2}$ across the absorbing section is given, using an approximation for the carrier density in a parabolic band [25] by

$$
\begin{aligned}
V_{2}= & V_{g}+V_{T}\left(\ln \left(\frac{N_{2}\left(N_{2}+N_{A}\right)}{N_{C} N_{V}}\right)\right. \\
& \left.+\frac{N_{2}}{2 \sqrt{2}}\left(\frac{1}{N_{C}}+\frac{1}{N_{V}}\right)+\frac{1}{2 \sqrt{2}} \frac{N_{A}}{N_{V}}\right) .
\end{aligned}
$$

In the expression above $V_{g}=1.46 \mathrm{~V}$ is the bandgap voltage, $V_{T}=25.9 \mathrm{mV}$ is the thermal voltage at room temperature $k T / q$, and $N_{C}=4.7 \times 10^{17} \mathrm{~cm}^{-3}$ and $N_{V}=7 \times 10^{18} \mathrm{~cm}^{-3}$ are the effective conduction band and valence band density of states, respectively. The calculated $I_{2}-V_{2}$ characteristic of the absorbing section obtained from the static solutions of (1) through (4) is shown in Fig. 13 and displays a region of negative differential resistance between $V_{2}=1.3 \mathrm{~V}$ and $V_{2}=1.4 \mathrm{~V}$. This negative differential resistance is obtained as the static solution of the conventional rate equations (1) through (4). Some of the difference between the measurements and the calculations is due to the neglected superluminescence, but it is believed that most is caused by the photoconductive effect, which is discussed in the next paragraph.

It has been assumed up to here that the gain and absorbing section are electrically insulated and that they only interact optically. However, there is an additional electrical coupling which consists of the following two parts. A constant parasitic resistance $R_{p}$ is due to the finite lateral conductance of the ptype top cladding layer, which has been made so large $\left(R_{p}=\right.$ $60 \mathrm{k} \Omega$ ) by doping this layer only slightly, that it can be ne-

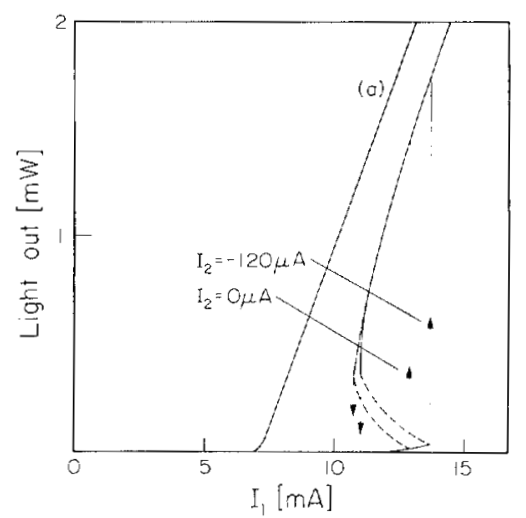

Fig. 11. Calculated static light-current characteristic. For homogeneous pumping: curve (a). For inhomogeneous pumping: curve (b) for $I_{2}=$ $-120 \mu \mathrm{A}$ and curve (c) for $I_{2}=0 \mu \mathrm{A}$. Parameters of the model are given in the text.

glected and a connection through carriers can be generated in the optical waveguide connecting gain and absorber section, which we will refer to as photoconductive effect. When the bistable laser is switched off, the resistance of this photoconductive path is very large $(\geqslant 200 \mathrm{k} \Omega)$, but if it is switched on, the carrier density within this section is increased to transparency level and the resistance is estimated to drop to about $2 \mathrm{k} \Omega$. This photoconductive effect adds constructively to the optoelectronic effect in the $I_{2}-V_{2}$ characteristic and is probably responsible for the main differences between measurements and calculation.

\section{Small Signal Analysis and Equivalent Circuit}

The small signal rate equations are obtained from (1) through (3) by substituting $N_{1}=N_{10}+n_{1}, N_{2}=N_{20}+n_{2}, P=P_{0}+p$, $I_{1}=I_{10}+i_{1}$ and $I_{2}=I_{20}+i_{2}$.

$$
\begin{aligned}
\frac{d n_{1}}{d t}= & \frac{i_{1}}{e V_{1}}-\left(\frac{1}{\tau_{1}}+A P_{0}\right) n_{1}-A\left(N_{10}-N_{t r}\right) p \\
\frac{d n_{2}}{d t}= & \frac{i_{2}}{e V_{2}}-\left(\frac{1}{\tau_{2}}+A P_{0}\right) n_{2}-A\left(N_{20}-N_{t r}\right) p \\
\frac{d p}{d t}= & \alpha_{1} n_{1}\left(\frac{\beta}{\tau_{1}}+A P_{0}\right)+\alpha_{2} n_{2}\left(\frac{\beta}{\tau_{2}}+A P_{0}\right) \\
& -A\left(N_{t r}+\frac{1}{A \tau_{p}}-\alpha_{1} N_{10}-\alpha_{2} N_{20}\right) p \\
\tau_{1}= & \frac{1}{B\left(2 N_{10}+N_{A}\right)} \\
\tau_{2}= & \frac{1}{B\left(2 N_{20}+N_{A}\right)} .
\end{aligned}
$$

In the equations above, $N_{10}, N_{20}$, and $P_{0}$ are the static solutions obtained from (1) through (3) for $I_{10}$ and $I_{20}$, and $n_{1}$, $n_{2}$, and $p$ are the small signal responses of the carrier densities of the gain section, absorber section, and photons, respectively, if the small signal currents $i_{1}$ and $i_{2}$ are injected into the gain and absorbing section.

Now consider the separate problem of the equivalent circuit 

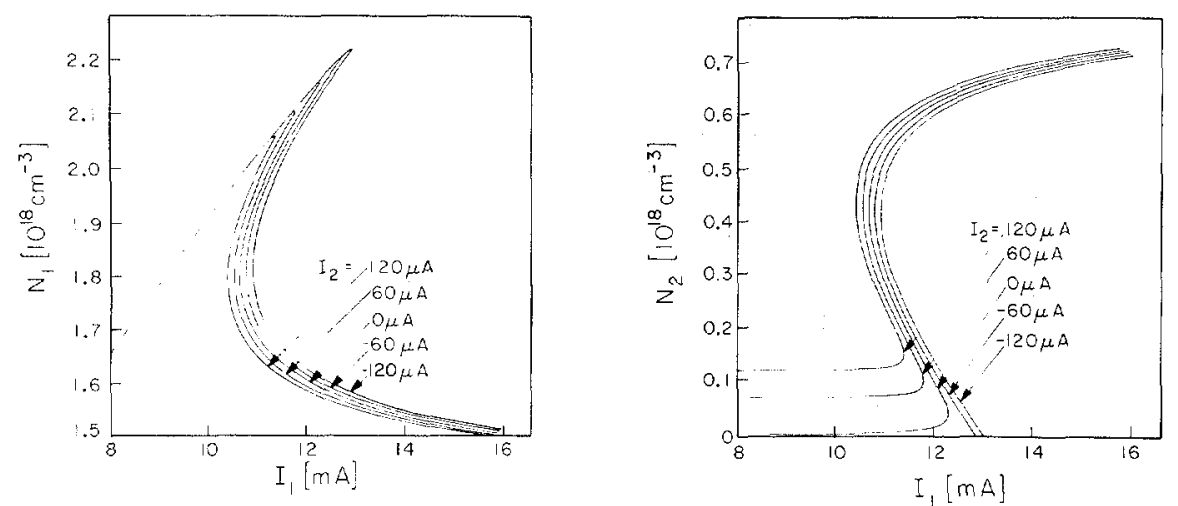

Fig. 12. Calculated carrier concentration in the gain section $\left(N_{1}\right)$ and in the absorber section $\left(N_{2}\right)$ as function of $I_{1}$, the current through the gain section for a set of different bias-currents $I_{2}$ through the absorber section.

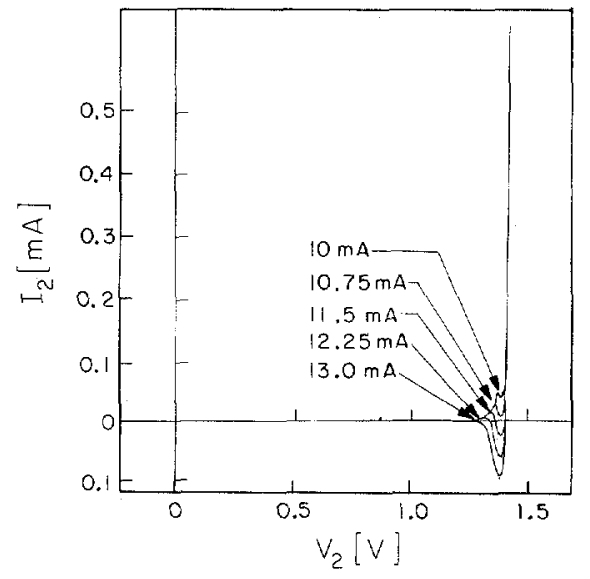

Fig. 13. Calculated current-voltage characteristic $\left(I_{2}-V_{2}\right)$ of the absorber section for different currents $I_{1}$ injected into the gain section.

as shown in Fig. 14(a). This circuit is described by the following set of circuit equations:

$$
\begin{aligned}
& \frac{d v_{1}}{d t}=\frac{i_{1}}{C_{1}}-\frac{v_{1}}{R_{1} C_{1}}-\frac{i_{L}}{C_{1}} \\
& \frac{d v_{2}}{d t}=\frac{i_{2}}{C_{2}}-\frac{v_{2}}{R_{2} C_{2}}+k \frac{i_{L}}{C_{2}} \\
& \frac{d i_{L}}{d t}=\frac{v_{1}}{L}+\frac{v_{2}}{N L}-\frac{R_{s e}}{L} i_{L}
\end{aligned}
$$

where $i_{1}$ and $v_{1}$ are the small signal current and voltage at the gain section and $i_{2}$ and $v_{2}$ are the corresponding variables for the absorber section. Setting $N_{1}=N_{10}+n_{1}$ and $N_{2}=N_{20}+$ $n_{2}$ in (4) yields the small signal relationships between carrier variation and voltage.

$$
\begin{aligned}
& v_{1}=m_{1} V_{T} \frac{n_{1}}{N_{10}} \quad m_{1}=\frac{2 N_{10}+N_{A}}{N_{10}+N_{A}}+\frac{N_{10}}{2 \sqrt{2}}\left(\frac{1}{N_{V}}+\frac{1}{N_{C}}\right) \\
& v_{2}=m_{2} V_{T} \frac{n_{2}}{N_{20}} \quad m_{2}=\frac{2 N_{20}+N_{A}}{N_{20}+N_{A}}+\frac{N_{20}}{2 \sqrt{2}}\left(\frac{1}{N_{V}}+\frac{1}{N_{C}}\right) .
\end{aligned}
$$

Comparing factors in (5)-(7) with (10)-(12) and using (13) and (14) leads to a proportionality between the current $i_{L}$ and the optical small signal power $p$.

$$
i_{L}=p A\left(N_{10}-N_{t r}\right) e V_{1}
$$

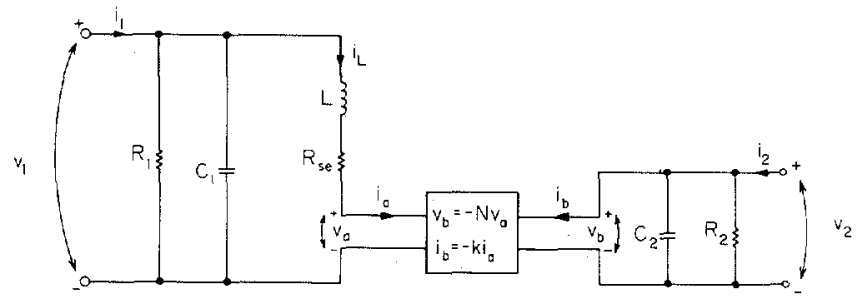

(a)

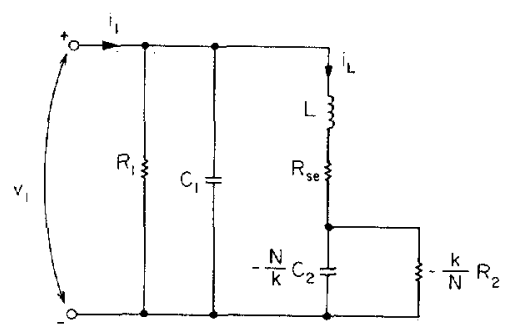

(b)

Fig. 14. Equivalent electrical small signal circuit of the two segment laser. The values of the elements are given in the text: (a) full equivalent circuit, (b) reduced equivalent circuit.

The following values for the elements of the equivalent circuit are also obtained.

$$
\begin{aligned}
C_{1} & =\frac{e V_{1} N_{10}}{m_{1} V_{T}} \\
R_{1} & =\frac{1}{C_{1}\left(\frac{1}{\tau_{1}}+A P_{0}\right)} \\
C_{2} & =\frac{e V_{2} N_{20}}{m_{2} V_{T}} \\
R_{2} & =\frac{1}{C_{2}\left(\frac{1}{\tau_{2}}+A P_{0}\right)} \\
L & =\frac{1}{C_{1} \alpha_{1} A\left(N_{10}-N_{t r}\right)\left(\frac{\beta}{\tau_{1}}+A P_{0}\right)} \\
R_{s e} & =L A\left(N_{t r}+\frac{1}{A \tau_{p}}-\alpha_{1} N_{10}-\alpha_{2} N_{20}\right)
\end{aligned}
$$




$$
\begin{aligned}
& k=\frac{\alpha_{2}\left(N_{t r}-N_{20}\right)}{\alpha_{1}\left(N_{10}-N_{t r}\right)} \\
& N=\frac{\alpha_{1} m_{2} N_{10}\left(\frac{\beta}{\tau_{1}}+A P_{0}\right)}{\alpha_{2} m_{1} N_{20}\left(\frac{\beta}{\tau_{2}}+A P_{0}\right)} .
\end{aligned}
$$

Fig. 14(b) shows the reduced equivalent circuit with the absorbing section represented by a negative resistance $-(k / N)$ $R_{2}$ with $(k / N)>0$. From this circuit it can be inferred that the useful small signal bandwidth of this gain element is limited to about $\left(1 / R_{2} C_{2}\right)=1 / \tau_{2}+A P_{0}$ (a few hundred megahertz) due to the shunting of $R_{2}$ by the capacitor $C_{2}$. The usefulness of the double contact laser as a negative resistance electrical circuit element [11], [26] is limited mainly by the contact resistance which makes it difficult to couple $-(k / N) R_{2}$ to an electrical circuit. Since the gain element $-(k / N) R_{2}$ is not frequency selective the device will pulsate with a frequency given by a frequency selective mechanism, such as the relaxation resonance (represented by $L$ and $C_{1}$ ) or the roundtrip time of an external optical cavity. A small signal analysis of a bistable laser in an external optical cavity shows that the negative resistance $-(k / N) R_{2}$ can be coupled very efficiently to the frequency selective mechanism of an external cavity. Measurements show that pulsations in an external optical cavity correspond to the roundtrip time and have harmonic contents up to the gigahertz range.

\section{Small Signal Analysis of the Critical Slowing Down}

In this paragraph we develop a small signal interpretation of the critical slowing down. The stability of the laser can be investigated by calculating the roots of the characteristic equation of the Laplace transform of (10)-(12) with $s$ as independent variable.

$$
\begin{aligned}
& \left(s+\frac{1}{R_{1} C_{1}}\right)\left\{\left(s+\frac{1}{R_{2} C_{2}}\right)\left(R_{s e}+s L\right)-\frac{k}{N} \frac{1}{C_{2}}\right\} \\
& +\frac{1}{C_{1}}\left(s+\frac{1}{R_{2} C_{2}}\right)=0 .
\end{aligned}
$$

The loci of the three roots in the complex s-plane are shown in Fig. 15 as a function of the power in the lasing mode. At low optical power all three roots are on the negative real axis and the laser is therefore stable. Increasing the power causes two roots to split off the negative real axis into the complex left half plane. The third root moves on the real axis towards the right, crosses the origin and remains on the positive real axis for biasing conditions where the light current characteristic has a negative slope. At high optical power densities this root returns to the negative real axis. At the transition points (switch-on $P=0.008 \mathrm{~mW}$ and switch-off $P=0.4 \mathrm{~mW}$ ) of the bistable laser this root of the characteristic equation is right at the origin. A root at the origin of the s-plane signifies an infinite long response time to any perturbation which results in a dead time. This corresponds to the critical slowing down [16] of the delay time at the transition points as observed.

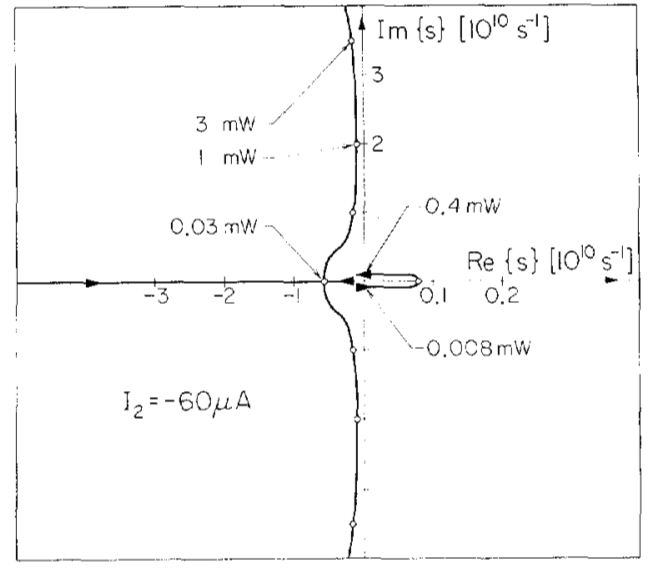

Fig. 15. Location of the three roots of the characteristic equation as function of the optical power emitted. One root is at the origin at the transition points, $P=0.008 \mathrm{~mW}$ and $P=0.4 \mathrm{~mW}$. Note that the scale of the positive real axis is expanded.

\section{External Optical Cavity}

The typical experimental arrangement of the device in an external optical cavity is shown in Fig. 16. Also shown is the light versus current through the gain section $I_{1}$ characteristic at a constant current through the absorber section $I_{2}$. A shift of the hysteresis under optical feedback is apparent. The amount of light fed back into the laser is estimated from the shift of the threshold current to be around 50 percent. To switch the bistable laser on and off by varying the optical feedback, it is necessary that the hysteresis with and without feedback do not overlap. This can be achieved with the specific combination of $I_{1}=29.5 \mathrm{~mA}$ and $I_{2}=-78 \mu \mathrm{A}$ [13] and the resulting light versus feedback characteristic is shown in Fig. 17. The voltage $V_{2}$ across the absorbing section (also shown in Fig. 17) depends on the feedback in a manner similar to that of the light output. The switching of the voltage $V_{2}$ through a change of optical feedback can be utilized, for example, in optical disk readout, in which a rotating disk carrying binary information as reflective spots is placed into the optical feedback path as shown in Fig. 18. The bistable laser serves as an integrated source detector combination. A larger flexibility in controlling the amount of optical feedback is achieved if one facet of the bistable laser is antireflection (AR) coated. Unlike the previous case with no AR-coating (Fig. 16), it is not necessary to maintain the bias currents $I_{1}$ and $I_{2}$ within a small range [13].

This application of the bistable laser as an optical stylus was simulated in our experiment by inserting a mechanical chopper in the external cavity. The measured voltage $V_{2}$, the output signal of the optical stylus, is shown in Fig. 19. The signal amplitude is very large $V_{p p}=400 \mathrm{mV}$, which is some three orders of magnitude larger than the signal of conventional self coupled detectors [27] which is only a few hundred microvolts. The slow rise (fall) in the voltage preceding the fast switch-on (switch-off) is caused by the finite transversal time of the chopper blade through the optical beam. The switching time is measured to be less than $70 \mathrm{~ns}$, consistent with that obtained by electrical switching of this bistable laser. Faster switching $(<20 \mathrm{~ns})$ can be achieved electrically by raising the switching 


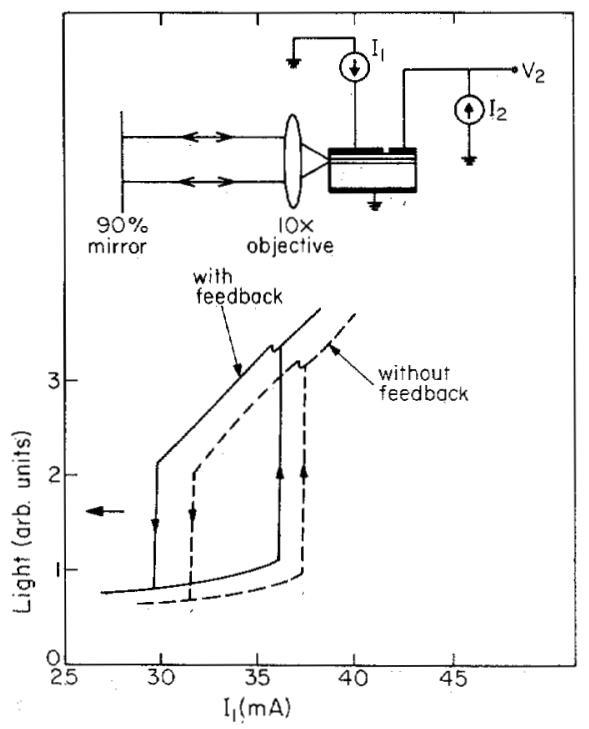

Fig. 16. Typical experimental setup of the bistable laser in an external optical cavity. Measured light versus current $I_{1}$ through the gain section characteristic without (solid line) and with (dashed line) optical feedback for $I_{2}=-110 \mu \mathrm{A}$. The amount of feedback is about 50 percent.

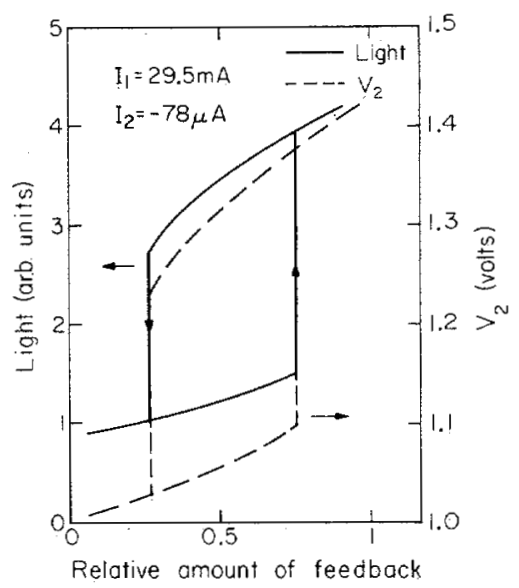

Fig. 17. Hysteresis in the light versus relative feedback characteristic (solid line). Hysteresis in the voltage $V_{2}$ across the absorber section versus relative feedback characteristic (dashed line). A relative feedback of 1 corresponds to a total feedback of 50 percent.

pulse overdrive, an option not available in optical feedback switching. Critical slowing down similar to that in electrical switching of up to several $\mu$ s is observed when the amount of feedback is just barely sufficient to cause switching. Using a bistable laser as an optical stylus in disk readout has the following advantages. The optical system can be kept simple, the signal to noise ratio is very good due to the optical schmidttrigger characteristic and the detector output signal is a few hundred millivolts large. The switching speed is compatible with audio-disk applications.

The results above were obtained with the device operating in the bistable regime, by driving the absorber section with a high impedance source. No pulsations can be observed within the detector bandwidth of $5 \mathrm{GHz}$. As discussed above, the laser can be made to pulsate and have a very narrow hysteresis when it is biased with a low impedance source. Without optical feedback the electrical and optical pulsations occur at the relaxa-
Integrated Laser-Detector for Video-Disk Readout

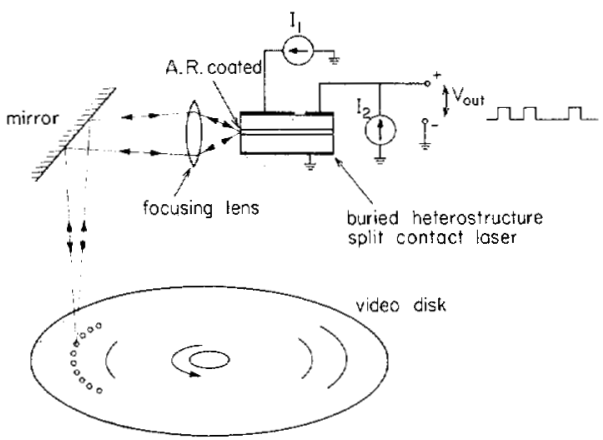

Fig. 18. Application of the bistable laser as an optical stylus. The bias currents $I_{1}$ and $I_{2}$ are held at a constant value and the bistable laser is switched on and off by the light reflected back from the optical disk. The detector output signal $V_{2}$ is very large, $V_{2 p p}=400 \mathrm{mV}$.

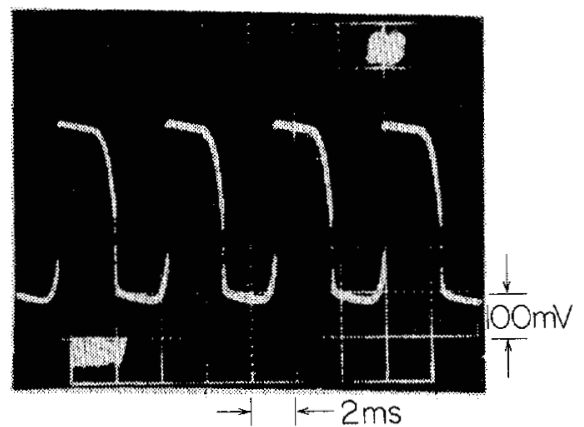

Fig. 19. Measured detector output signal $V_{2}$ of the optical stylus shown in Fig. 18. The optical feedback is modulated by a rotating chopper disk in the optical feedback path. Horizontal: $2 \mathrm{~ms} / \mathrm{div}$, vertical: $100 \mathrm{mV} / \mathrm{dv}$.

tion resonance. With optical feedback the external cavity becomes the dominant frequency selective element and the laser is observed to pulsate at the roundtrip time of the external optical cavity. Note that such pulsations do not occur when the absorber section is driven with a current source in marked contrast to the results obtained using aged or damaged lasers [28]-[30]. This indicates that even though the intrinsic absorption of the semiconductor laser does not saturate more easily than the gain, the presence of inhomogeneous excitation in GaAs lasers can still produce pulsations. This can be understood by taking the electrical aspect of the injection laser into account as pointed out above.

\section{Conclusions}

We have found that double contact injection lasers can be made to display a large hysteresis in the light-current characteristic when the gain and absorber section are well insulated from each other. A negative differential resistance, which is optoelectronic in origin, is observed across the absorber section. Depending on the load impedance biasing the absorber section, this negative resistance can lead to a) bistability with a very large hysteresis in the light-current characteristic without self pulsations or b) a narrow hysteresis (or a light jump) with self pulsations at microwave frequencies. These results indicate that in the absence of side effects, such as local heating [31] or proton bombardment induced defects [29], the intrinsic 
absorption of GaAs does not saturate more easily than the gain and therefore will not produce pulsations due to repetitively $Q$-switching [31]. The presence of inhomogeneous excitation caused by nonuniform contacts or centers of nonradiative recombination can however produce light-jumps and pulsations through a different mechanism involving the electrical aspect of the device as illustrated in Sections III and V.

The dynamic switching characteristic of the bistable injection laser is investigated in some detail. While the switch-off is usually fast, the switch-on delay shows critical slowing down. The delay can be reduced to less than $20 \mathrm{~ns}$ by increasing the trigger pulse overdrive. Switching delays between a few and a few hundred nanoseconds and power delay products between a few and a few hundred picojoules are obtained depending on the switching mechanism and the trigger pulse overdrive.

A simple model is analyzed which displays the main features of a semiconductor laser with inhomogeneous current injection, namely a large hysteresis in the light current characteristic and a negative differential resistance across the absorber section. A small signal analysis of this model leads to an equivalent electrical circuit which clarifies the frequency limitations of this negative differential resistance. This analysis leads also to a small signal interpretation of the observed critical slowing down at the transition points.

Experiments on interactions of this laser with an external optical cavity show that the behavior depends on the load impedance at the absorber section. A large load resistance produces bistability and a small one produces pulsations at the round trip time of the optical cavity with harmonics up to several $\mathrm{GHz}$. In bistable operation, the switching can also be achieved by varying the amount of optical feedback. The associated switching of the voltage across the absorber section can be utilized in digital disk readout. We showed that a bistable laser with an AR coating on one facet is even more suitable for this task. The switching speed is compatible with the application of this device as an optical stylus for audiodisk readout.

\section{REFERENCES}

[1] G. J. Lasher, "Analysis of a proposed bistable injection laser," Solid-State Electron., vol. 7, pp. 707-716, Oct. 1964.

[2] M. I. Nathan, J. C. Marinace, R. F. Rutz, A. E. Michel, and G. J. Lasher, "GaAs injection laser with novel mode control and switching properties," J. Appl. Phys, vol. 36, pp. 473-480, Feb. 1965.

[3] H. Kawaguchi and G. Iwane, "Bistable operation in semiconductor lasers with inhomogeneous excitation," Electron. Lett., vol. 17 , pp. 167-168, Feb. 1981.

[4] H. Kawaguchi, "Bistable operation of semiconductor lasers by optical injection," Electron. Lett., vol. 17, pp. 741-742, Oct. 1981.

[5] J. K. Carney and C. G. Fonstad, "Double-heterojunction laser diodes with multiply segmented contacts," Appl. Phys. Lett., vol. 38, pp. 303-305, Mar. 1981.

[6] Ch. Harder, K. Y. Lau, and A. Yariv, "Bistability and pulsations in CW semiconductor lasers with a controlled amount of saturable absorption," Appl. Phys. Lett., vol. 39, pp. 382-384, Sept. 1981.

[7] T. P. Lee and R.H.R. Roldan, "Repetitively Q-switched light pulses from $\mathrm{GaAs}$ with tandem double-section stripe geometry," IEEE J. Quantum Electron, vol. QE-6, pp. 339-352, June 1970.

181 N. G. Basov, "0-1-dynamics of injection lasers," IEEE J. Quantum Electron., vol. QE-4, pp. 855-964, Nov. 1968.

[9] H. Ito, N. Onodera, K. Gen-ei, and H. Inaba, "Self- $Q$-switched picosecond generation with tandem-type AlGaAs TJS laser," Electron. Lett., vol. 17, pp. 15-17, Jan. 1981.
[10] K. Hanamitsu, T. Fujiwara, and M. Takusagawa, "Repetitive pulsating stripe geometry GaAlAs double-heterostructure lasers with a stripe by a shallow $\mathrm{Zn}$ diffusion," Appl. Phys. Lett., vol. 39, pp. 14-16, July 1981.

[11] Ch. Harder, K. Y. Lau, and A. Yariv, "Bistability and negative resistance in semiconductor lasers," Appl. Phys. Lett., vol. 40, pp. 124-126, Jan. 1982.

[12] K. Y. Lau, Ch. Harder, and A. Yariv, "Dynamical switching characteristic of a bistable injection laser," Appl. Phys. Lett., vol. 40, pp. 198-200, Feb. 1982.

[13] K. Y. Lau, Ch. Harder, and A. Yariv, "Interaction of a bistable injection laser with an external optical cavity," Appl. Phys. Lett., vol. 40, pp. 369-371, Mar. 1982.

[14] R. W. Dixon and W. B. Joyce, "A possible model for sustained oscillations (pulsations) in ( $\mathrm{Al}, \mathrm{Ga}) \mathrm{As}$ double-heterostructure lasers," IEEE J. Quantum Electron., vol. QE-15, pp. 470-474, June 1979.

[15] T. Ohmi and S. Yamazaki, "A limitation on the rate of pulsations of junction lasers due to the repetitively Q-switched mechanism," IEEE J. Quantum Electron., vol. QE-9, pp. 366-374, Feb. 1973.

[16] R. Bonifacio and P. Meystre, "Critical slowing down in optical bistability," Opt. Commun., vol. 29, pp. 131-134, Apr. 1979

[17] E. Garmire, J. H. Marburger, S. D. Allen, and H. G. Winful, "Transient response of hybrid bistable optical devices," Appl. Phys. Lett., vol. 34, pp. 374-376, Mar. 1979.

[18] N. Bar-Chaim, J. Katz, I. Ury, and A. Yariv, "Buried heterostructure AlGaAs lasers on semi-insulating substrates," Electron. Lett., vol. 17, pp. 108-109, Feb. 1981

[19] C. H. Henry, R. A. Logan, and K. A. Bertness, "Spectral dependence of the change in refractive index due to carrier injection in GaAs lasers," J. Appl. Phys, vol. 52, pp. 4457-4461, July 1981 .

[20] J. F. Scott, M. Sargent III, C. D. Cantrell, "Laser-phase transition analogy: Application to first order phase transitions," Opt. Commun., vol. 15, pp. 13-16, 1975 .

[21] L. A. Lugiato, P. Mandel, S. T. Dembinski, and A. Kossakowski, "Semiclassical and quantum theories of bistability in lasers containing saturable absorbers," Phys. Rev. A, vol. 18, pp. 238-254, 1978.

[22] H. Kressel and J. K. Butler, Semiconductor Lasers and Heterojunction LEDs. New York: Academic, 1977.

[23] C. H. Henry, R. A. Logan, and F. R. Merritt, "Measurement of gain and absorption spectra in $\mathrm{AlGaAs}$ buried heterostructure lasers," J. Appl. Phys., vol. 51, pp. 3042-3050, June 1980.

[24] D. Marcuse and F. R. Nash, "Computer model of an injection laser with asymmetrical gain distribution," IEEE J. Quantum Electron., vol. QE-18, pp. 30-43, Jan. 1982.

[25] W. B. Joyce and R. W. Dixon, "Analytic approximations for the Fermi energy of an ideal Fermi gas," Appl. Phys. Lett., vol. 31, pp. 354-356, Sept. 1977.

[26] T. L. Paoli, "Electrical interactions of a superlinear laser diode with its external circuit," IFEE J. Quantum Electron., vol. QE16, pp. 1248-1250, Nov. 1980.

[27] Y. Mitsuhashi, J. Shimada, and S. Mitsutsuka, "Voltage change across the self-coupled semiconductor laser," IEEE J. Quantum Electron., vol. QE-17, pp. 1216-1225, July 1981.

[28] K. Y. Lau, L. Figueroa, and A. Yariv, "Generation and quenching of intensity pulsations in semiconductor lasers coupled to external cavities," IEEE J. Quantum Electron., vol. QE-16, pp. 1329-1336, Dec. 1980

[29] J. P. van der Ziel, W. T. Tsang, R. A. Logan, and W. M. Augustyniak, "Pulsating output of a separate confinement buried optical guide lasers due to the deliberate introduction of saturable loss," Appl. Phys. Lett., vol. 39, pp. 376-378, Sept. 1981.

[30] J. P. van der Ziel, W. T. Tsang, R. A. Logan, R. M. Mikulyak, and W. M. Augustyniak, "Subpicosecond pulses from passively modelocked GaAs buried optical guide semiconductor lasers," Appl. Phys. Lett., vol. 39, pp. 525-527, Oct. 1981.

[31] C. H. Henry, "Theory of defect-induced pulsations in semiconductor injection lasers," $J$. Appl. Phys., vol. 51, pp. 3051-3061, June 1980.

Christoph Harder, for a photograph and biography, see p. 337 of the March 1982 issue of this Journal. 


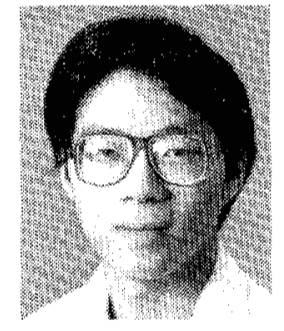

Kam Y. Lau (M'78) was born in Hong Kong in October 1956. He received a simultaneous B.S. and M.S. degree in electrical engineering in June 1978, and the Ph.D. degree in electrical engineering in June 1981, all from California Institute of Technology, Pasadena.

He worked at Bell Laboratories, Holmdel, NJ, on optical modulators during the summers of 1977 and 1978. While in graduate school, he worked part time at the Jet Propulsion Laboratory, Pasadena, CA, where he was responsible for development of fiber optic systems for specialized applications in the NASA Deep Space Network. He received a distinguished achieve- ment award from NASA in 1981. He was a Research Fellow at California Institute of Technology since June 1981, until he joined the Ortel Corporation, Alhambra, CA, in October 1981, where he is currently continuing work on semiconductor lasers and related technologies.

Dr. Lau is a member of the Optical Society of America.

Amnon Yariv (S'56-M'59-F'70), for a photograph and biography, see p. 337 of the March 1982 issue of this Journal.

\title{
Quantum Theory of the Complex Dielectric Constant of Free Carriers in Polar Semiconductors
}

\author{
BARBARA JENSEN
}

\begin{abstract}
The optical constants and reflectivity of a semiconductor are known as functions of the real and imaginary parts of the complex dielectric constant. The imaginary part of the complex dielectric constant $e_{2}$ is proportional to the optical conductivity, which has recently been calculated from the quantum density matrix equation of motion. The expression obtained for $e_{2}$ reduces to the Drude result, as obtained from the quasi-classical Boltzmann transport equation, in the limit of low frequencies and elastic scattering mechanisms, and to the quantum result found using time dependent perturbation theory in the limit of high frequencies.

This paper derives the real part of the complex dielectric constant $e_{1}$ for a III-V or II-VI semiconductor with the band structure of the Kane theory, using the quantum density matrix method. The relation of $e_{1}$ to the second order perturbation energy of the system is shown, and the reflectivity is a minimum when the second order perturbation energy vanishes. The quantum calculation for $e_{1}$ gives approximately the same result as the Drude theory, except near the fundamental absorption edge, and reduces to the Drude result at low frequencies.

Using the complex dielectric constant, the real and imaginary parts of the complex refractive index, the skin depth, the surface impedance, and the reflectivity are found. The plasma resonance is examined. The surface impedance and the skin depth are shown to reduce to the usual classical result in the limit that $e_{1}=0$ and $w r<<1$, where $w$ is the angular frequency of the applied field and $\tau$ is the electron scattering time.
\end{abstract}

\section{INTRODUCTION}

$\mathrm{T}$ HE infrared absorption spectrum of free carriers in a semiconductor can be described in terms of a complex dielectric constant.

$$
\begin{aligned}
& \epsilon=e_{1}-i e_{2}=e e^{-i \theta} \\
& e=\left(e_{1}^{2}+e_{2}^{2}\right)^{1 / 2}, \quad \theta=\tan ^{-1} e_{2} / e_{1} .
\end{aligned}
$$

Manuscript received March 22, 1982. This work was supported by the Department of Energy under Contract DE-AC02-79ER10444.A000.

The author is with the Department of Physics, Boston University, Boston, MA 02215.
The real part of the complex dielectric constant $e_{1}$, and the imaginary part $e_{2}$, are functions of the complex refractive index $N$ as follows:

$$
\begin{aligned}
N & =n-i K, \quad e=n^{2}+K^{2} \\
e_{1} & =n^{2}-K^{2} \\
e_{2} & =2 n K .
\end{aligned}
$$

The imaginary part of the dielectric constant is proportional to the optical conductivity $\sigma$ and to the absorption coefficient $\alpha$.

$$
e_{2}=4 \pi \sigma / w=(c / w) n \alpha=2 n K
$$

where $w$ is the angular frequency. The optical conductivity, which gives the relation between the induced current and the applied electric field is calculated from the Boltzmann transport equation in the quasi-classical treatment. In the relaxation time approximation, one obtains the Drude result

$$
\begin{aligned}
\sigma & =\sigma_{0} /\left(1+w^{2} \tau^{2}\right) \rightarrow\left[\begin{array}{ll}
\sigma_{0}, & w \tau<<1 \\
\sigma_{0} / w^{2} \tau^{2}, & w \tau \gg 1
\end{array}\right. \\
\sigma_{0} & =n_{e} e \mu=n_{e} e^{2} \tau / m^{*}
\end{aligned}
$$

where $r$ is the electron scattering time, $\mu$ is the mobility, and $m^{*}$ is the effective electron mass.

The experimental procedure is to measure the reflectivity

$$
r=(n-1)^{2}+K^{2} /(n+1)^{2}+K^{2}
$$

and the transmission

$$
T=(1-r)^{2} e^{-\alpha d} /\left(1-r^{2} e^{-2 \alpha d}\right)
$$

where $d$ is the sample thickness, and to evaluate the real part of the refractive index $n$, the extinction coefficient $K$, and the absorption coefficient $\alpha$, using the preceding relations. 